\title{
Tendências das publicações brasileiras sobre a formação de professores indígenas em ciências da natureza
}

Trends of Brazilian publications on indigenous teachers training in the natural sciences

Silvana Costa Santa Rosa ${ }^{1}$

Edinéia Tavares Lopes ${ }^{2}$

\section{Resumo}

A partir da Constituição Federal de 1988, inicia-se a discussão sobre um novo formato de escola indígena. Para esse novo formato de escola, surge a necessidade da formação de professores indígenas, para atender a carência desse profissional, na educação básica. Esse estudo teve como objetivo realizar um levantamento das publicações nacionais, dos últimos quinze anos, sobre a formação de professores indígenas em Ciências da Natureza. Para selecionar teses, dissertações e artigos nacionais, relacionados com a temática em estudo, foram realizadas buscas nos sítios da Biblioteca Digital de Teses e Dissertações (BDTD), do Scientific Eletronic Library Online (Scielo) e do periódico da Coordenação de Aperfeiçoamento de Pessoal de Ensino Superior (CAPES). A partir das leituras dos resumos, foram selecionadas as produções que apresentavam relação direta com a temática investigada. Realizamos a leitura das produções, identificando o quantitativo, ano, estados, regiões, instituições vinculadas, nível, contextos investigativos e o que anunciam. Foram encontrados 58 trabalhos, sendo esses, 21 teses, 17 dissertações e 20 artigos. Desse total, apenas 2 trabalhos apresentam relação direta com o tema, suas discussões estão voltadas para as tensões e conflitos decorrentes do encontro entre saberes indígenas e os saberes provenientes da Ciência Moderna, assim como da diversidade cultural presente na formação de professores indígenas. Compreendemos, que a formação de professores indígenas em Ciências da Natureza, exerce papel chave para que a área de Ensino de Ciências colabore com os projetos de autossustentação e autodeterminação dos povos indígenas, daí a importância de se conhecer como está a produção científica sobre o tema. Verificamos que o campo de formação de professores indígenas é novo no Brasil e, mais especificamente, as investigações sobre a formação de professores indígenas em Ciências da Natureza.

Palavras chave: formação de professores indígenas; licenciaturas interculturais.

\section{Abstract}

From the Federal Constitution of 1988, initiates it quarrel on a new format of aboriginal school. For this new format of school, the necessity appears of the formation of aboriginal

\footnotetext{
${ }^{1}$ Universidade Federal de Sergipe | silvana.csr@gmail.com

${ }^{2}$ Universidade Federal de Sergipe | edineia.ufs@gmail.com
} 
professors, to take care of the lack of this professional, in the basic education. This study it had as objective to carry through a survey of national publications, of last the fifteen years, on the formation of aboriginal professors in Sciences of the Nature. To select national thesis, dissertations and articles, related with the thematic one in study, searches in the small farms of the Digital Library of Thesis and dissertations had been carried through (BDTD), of the Scientific Eletronic Library Online (Scielo) and of periodic of the Coordination of Perfectioning of Staff of Higher education (CAPES). From the readings of the summaries, the productions had been selected that presented investigated direct relation with the thematic one. We carry through the reading of the productions, identifying quantitative, the year, states, tied regions, institutions, research contexts, contexts and what they announce. 58 works had been found, being these, 21 thesis, 17 dissertations and 20 articles. Of this total, only 2 works present direct relation with the subject, its quarrels are come back toward the tensions and consequence conflicts of the meeting between knowing aboriginals and knowing them proceeding from Modern Science, as well as of the present cultural diversity in the formation of aboriginal professors. We understand, that the formation of aboriginal professors in Sciences of the Nature, exerts paper key so that the area of Education of Sciences collaborates with the projects of self-suppression and self-determination of the aboriginal peoples, from there the importance of if knowing as it is the scientific production on the subject. We verify that the field of formation of aboriginal professors is new in Brazil and, more specifically, the inquiries on the formation of aboriginal professors in Sciences of the Nature.

Keywords: indigenous teacher training; intercultural education.

\section{Introdução}

As mudanças na sociedade contemporânea refletem diretamente na educação escolar e na formação de professores. Decerto, o professor exerce uma função importante na sociedade e no processo educativo, pois, ele é um profissional formador de opiniões e, de maneira muito significativa, contribui para a formação das pessoas.

Decorre daí o destaque da temática formação de professores nos debates e políticas públicas acerca da educação, pois se entende que a formação docente - inicial e continuada - pode estar diretamente relacionada com sua prática pedagógica. Nesse sentido, existe um consenso que sem investimentos na formação de professores não se pode alcançar verdadeiras transformações no processo educacional (FERNANDES, 2009).

Sobre essa formação, muitas iniciativas importantes podem ser visualizadas no cenário nacional, que buscam fortalecer a formação de professores, porém, ainda é possível verificar antigos problemas como a desvalorização profissional e a "precarização" do trabalho. Somam-se a isso, novos desafios como a terceirização da mão de obra docente, os frequentes questionamentos acerca da função do professor na sala de aula e a diversidade cultural brasileira (Fernandes, 2009).

Nesse cenário talvez seja ainda mais desafiador a formação de professores indígenas, pois ela, considerada como primordial para a consolidação de uma Educação Escolar 
Indígena ${ }^{3}(E E I)$, deva se pautar pelos princípios da especificidade, do bilinguismo e da interculturalidade, previstos na Constituição Federal (BRASIL, 1988) e no Referencial Curricular Nacional para as Escolas Indígenas (RCNEI), (Brasil, 2002).

Essa formação é uma tarefa complexa, já que em cada local do país se encontra uma solução diferente e não existe um único modelo a ser adotado, devido a diversidade cultural, linguística, histórica, política, de formação e escolarização vividos pelos professores indígenas e por suas comunidades (GRUPIONI, 2003).

Sobre isso, Lopes et al (2017) afirmam que a efetivação do projeto de EEl carece de parcerias institucionais nos quais a formação dos professores indígenas em cursos específicos - com currículo diferenciado -, de fato, atenda às novas diretrizes para essa modalidade e as demandas colocadas pelos mais de 305 povos indígenas brasileiros. Apontam que é imprescindível a inclusão, de fato, das pedagogias indígenas nessa modalidade de ensino, aliadas aos conhecimentos acadêmicos.

Lopes (et al, 2017) acrescentam que é preciso considerar, dentre outros desafios, que ainda existe um expressivo número de professores indígenas que atuam nas escolas das aldeias sem formação específica e também a necessidade de concurso diferenciado. Os autores ainda acrescentam que, sem dúvida, foram significativas as conquistas educacionais dos povos indígenas brasileiros nas últimas décadas - pós Constituição Federal de $1988^{4}$-, mas há carência na produção científica nacional, sobretudo no que diz respeito a área das Ciências da Natureza ${ }^{5}$ (CN) na EEl (LOPES, 2015).

A nosso ver, os estudos sobre a formação de professores indígenas são indispensáveis para efetivação da EEl em nosso país, com destaque para a região Norte, onde vivem cerca de $60 \%$ dos povos indígenas brasileiros; e a escola e os professores, historicamente, são referências para essas comunidades, configurando-se como eixos chave para a afirmação e valorização das tradições e identidades indígenas (CAVALCANTE, 2003).

Desse modo, este artigo ${ }^{6}$ tem objetivo de apresentar o mapeamento das publicações nacionais, dos últimos quinze anos, sobre a formação de professores indígenas em Ciências da Natureza, esse trabalho favorece a compreensão, do que anunciam os trabalhos já realizados sobre a temática em estudo.

${ }^{3}$ Collet (2006) explica que é proposto por autores como (Meliá (1970) e Lopes (1980) que EEl é a denominação dada a educação oferecida formalmente na escola, enquanto Educação Indígena, são as práticas tradicionais, de socialização e transmissão de conhecimentos próprios a cada sociedade indígena.

${ }^{4}$ A constituição brasileira de 1988, demarcou uma nova fase no que diz respeito aos direitos dos povos indígenas desse país, conferindo-lhes garantias mínimas que dizem respeito, dentre outros, a demarcação dos territórios, a organização social, as línguas maternas e aos processos próprios de aprendizagem (LOPES et al, 2017).

${ }^{5}$ Compreendemos como Ciências da Natureza a área de conhecimento do Ensino Médio que integra a Física, a Química e a Biologia como ciências que têm em comum a investigação da natureza e compartilham linguagens para a representação e sistematização do conhecimento de fenômenos ou processos naturais e tecnológicos. (Brasil, 2006).

${ }^{6}$ Esse artigo é parte constituinte de um contexto maior, que se trata de uma pesquisa de mestrado sobre a formação de professores indígenas em ciências da natureza na região Norte do país. Essa pesquisa, entre outras, está sendo desenvolvida no âmbito do projeto intitulado "Educação em Ciências na Perspectiva da Educação Intercultural: Investigando e Construindo Possibilidades", que está sendo financiado pelo Conselho Nacional de Desenvolvimento Científico e Tecnológico (CNPq). 


\section{Percurso metodológico}

Para selecionar teses, dissertações e artigos nacionais, relacionados com a temática em estudo, publicados dentro do recorte temporal de 2002 a 2017, foram realizadas buscas nos sítios da Biblioteca Digital de Teses e Dissertações (BDTD), do Scientific Eletronic Library Online (Scielo) e do periódico da Coordenação de Aperfeiçoamento de Pessoal de Ensino Superior (CAPES). Buscando identificar o máximo possível de produções científicas, foram utilizados vários termos que se relacionavam a formação de professores indígenas para o ensino de CN (Quadro 1).

\begin{tabular}{l|l}
$\begin{array}{l}\text { Quadro 01: Termos usados no levantamento das publicações nacionais } \\
\text { sobre a formação de professores indígenas para o ensino de CN } \\
\text { realizado, nos sites da CAPES, BDTD e SCIELO, em } 2017 .\end{array}$ \\
\hline Primeiro termo & Segundo termo \\
\hline \multirow{4}{*}{$\begin{array}{l}\text { "Formação de professores } \\
\text { indígenas" }\end{array}$} & "Ensino de Ciências" \\
\cline { 2 - 2 } & "Ensino de Física" \\
\cline { 2 - 2 } & "Ensino de Biologia" \\
\cline { 2 - 2 } & "Ensino de Química" \\
\cline { 2 - 2 } & "Educação Intercultural" \\
\hline \multirow{5}{*}{$\begin{array}{l}\text { "Professores Indígenas" } \\
\end{array}$} & "Ensino de Ciências" \\
\cline { 2 - 2 } & "Ensino de Física" \\
\cline { 2 - 2 } & "Ensino de Biologia" \\
\cline { 2 - 2 } & "Ensino de Química" \\
\cline { 2 - 2 } & "Educação Intercultural' \\
\hline
\end{tabular}

A partir das leituras dos resumos selecionamos as produções que apresentavam relação direta com a temática investigada e realizamos a leitura das produções identificando o quantitativo, ano, estados, regiões, instituições vinculadas, nível, contextos investigativos e o que anunciam, conforme relatado a seguir.

\section{Formação de professores indígenas nas publicações brasileiras: reflexões iniciais}

A partir da coleta, no total, obtivemos 63 trabalhos, sendo que, dentre eles, 1 trabalho não apresentava discussões voltadas para a formação do professor indígena e 4 trabalhos foram localizados duas vezes no sítio da BDTD. De tal modo, o primeiro trabalho foi excluído da análise e os 4 que estavam duplicados no sítio da BDTD, naturalmente, foi contabilizado apenas uma vez, totalizando, portanto, a amostra final, com 58 trabalhos, sendo esses, 21 teses, 17 dissertações e 20 artigos. Os trabalhos são de instituições de ensino superior pública (federais (48), estaduais (02) e municipais (01)) comunitárias (03) e particulares (10). Esses trabalhos foram produzidos entre 2003 e 2017.

Os artigos foram desenvolvidos por pesquisadores vinculados a instituições do Amapá (5\%), Amazonas (10\%), Mato Grosso (15\%), Mato Grosso do Sul (5\%), Minas Gerais (5\%) Paraná (15\%), Goiás (10\%), Santa Catarina (15\%), Rio Grande do Sul (5\%) e São Paulo (15\%). 
As dissertações encontradas foram em maiores percentuais, desenvolvidas no estado do Rio Grande do Sul com 23,53\%, seguido dos estados do Pará, Minas Gerais, São Paulo, Amazonas e Mato Grosso do Sul com 11, 73\% cada e dos estados de Goiás, Sergipe, Santa Catarina com 5,88\% cada um. Quanto as teses, o estado de São Paulo apresentou maior quantidade de produção, com 61,90\%, em seguida os estados do Amazonas e Bahia com 9,53\% cada e dos estados do Paraná, Rio Grande do Sul, Santa Catarina e Minas Gerais com 4,76\% cada um (Tabela 01).

Tabela 01: Publicações brasileiras, encontradas em 2017 nos sites da CAPES, BDTD e SCIELO, sobre a formação de professores indígenas para o Ensino de CN por estado da federação.

\begin{tabular}{l|l|l|l|c}
\hline \multirow{2}{*}{ Estados } & \multicolumn{4}{l}{ Tipo de produção } \\
\cline { 2 - 5 } & Artigo & Dissertação & Tese & Totais \\
\hline AM & $02(10 \%)$ & $02(11,76 \%)$ & $02(9,52 \%)$ & 06 \\
\hline AP & $01(5 \%)$ & 00 & 00 & 01 \\
\hline BA & 00 & 00 & $02(9,52 \%)$ & 02 \\
\hline GO & $02(10 \%)$ & $01(5,88 \%)$ & 00 & 03 \\
\hline MG & $01(5 \%)$ & $02(11,76 \%)$ & $01(4,76 \%)$ & 04 \\
\hline MS & $01(5 \%)$ & $02(11,76 \%)$ & 00 & 03 \\
\hline MT & $03(15 \%)$ & 00 & 00 & 03 \\
\hline PA & 00 & $02(11,76 \%)$ & 00 & 02 \\
\hline PR & $03(15 \%)$ & 00 & $01(4,76 \%)$ & 04 \\
\hline RS & $01(5 \%)$ & $04(23,53 \%)$ & $01(4,76 \%)$ & 06 \\
\hline SE & 00 & $01(5,88 \%)$ & 00 & 01 \\
\hline SC & $03(15 \%)$ & $01(5,88 \%)$ & $01(4,76 \%)$ & 05 \\
\hline SP & $03(15 \%)$ & $02(11,76 \%)$ & $13(61,90 \%)$ & 18 \\
\hline Totais & $20(100 \%)$ & $17(100 \%)$ & $21(100 \%)$ & 58 \\
\hline
\end{tabular}

Em relação a produção dos artigos, as instituições dos estados do Mato Grosso, Paraná, São Paulo e Santa Catarina apresentaram maiores percentuais, seguidos por Bahia e Amazonas, os menores percentuais foram apresentados por Rio Grande do Sul, Amapá, Mato Grosso do Sul e Minas Gerais. No que se refere às dissertações o estado do Rio Grande do Sul apresentou a maior quantidade, a segunda maior produção está nos estados do Mato Grosso do Sul, Amazonas, São Paulo, Pará e Minas Gerais, seguido por Goiás, Santa Catarina e Sergipe com menores percentuais. Sobre as teses, o estado de São Paulo se destacou, apresentando 61,90\% das produções, seguido pelos estados da Bahia e Amazonas com 9,52\% cada. Paraná, Rio Grande do Sul, Santa Catarina e Minas Gerais apresentaram o menor percentual 4,76\% cada um.

Agrupando os artigos, dissertações e teses constatamos que o estado de São Paulo apresentou maior número de produções, sendo 31,03\%, seguido do estado do Rio Grande do Sul e Amazonas com 10,34\%. O estado de Santa Catarina apresentou 8,62\%. Os estados de Minas Gerais e Paraná apresentaram 6,89\%. Mato Grosso, Mato Grosso do Sul e Goiás apresentaram o quinto maior percentual que foi de 5,17\%. Bahia e Pará apresentaram 3,45\%, Amapá e Sergipe apresentaram 1,72\%. Portanto, as instituições dos estados de SP, RS, AM e SC têm se destacado nas produções científicas que abordam a formação de professores indígenas. 
Quando agrupamos essas produções por região do País, verificamos que estão concentradas, na sua maioria, na região Sudeste (37,9\%), seguida da região sul $(25,86 \%)$. Centro Oeste e Norte apresentam o mesmo quantitativo (15,51\%), o menor número de produções está na região nordeste (5,17\%).

Em continuidade, se organizou as teses, dissertações e artigos em cinco grandes grupos, conforme o tema investigado: 1 - Ensino e Aprendizagem na formação de professores indígenas; 2 - Avaliação de cursos de formação de professores indígenas; 3 Desenvolvimento de perspectivas educacionais e áreas de estudo na formação de professores indígenas; 4 - Avaliação de Políticas Públicas e Documentos Oficiais para a formação de professores indígenas; 5- Formação de professor indígena para o Ensino de Ciências da Natureza.

Dos 58 trabalhos encontrados, 22 foram organizados em G1 - Ensino e Aprendizagem na formação de professores indígenas, 14 foram agrupados em G2 - Avaliação de cursos de formação de professores indígenas, 12 foram agrupados em G3 - Desenvolvimento de perspectivas educacionais e áreas de estudo na formação de professores indígenas, 08 agrupados em G4- Avaliação de Políticas Públicas e Documentos Oficiais para a formação de professores indígenas e 02 foram agrupados em G5- Formação de professor indígena para o Ensino de Ciências da Natureza (Quadro 2).

Quadro 02: Publicações brasileiras encontradas nos sites da CAPES, BDTD e SCIELO, em 2017, que abordam a formação de professores indígenas, organizadas em grupos de acordo com a abordagem apresentadas nos texto.

\begin{tabular}{|c|c|c|}
\hline Grupo & Subtotais & Subtotais \\
\hline \multirow{3}{*}{$\begin{array}{l}\text { G1 - Ensino e aprendizagem na formação de professores } \\
\text { indígenas }\end{array}$} & $08 \mathrm{~A}$ & \multirow{3}{*}{22} \\
\hline & $04 \mathrm{D}$ & \\
\hline & $10 \mathrm{~T}$ & \\
\hline \multirow{3}{*}{$\begin{array}{l}\text { G2 - Avaliação de Cursos de Formação de Professores } \\
\text { Indígenas }\end{array}$} & $08 \mathrm{~A}$ & \multirow{3}{*}{14} \\
\hline & $04 \mathrm{D}$ & \\
\hline & $10 \mathrm{~T}$ & \\
\hline \multirow{3}{*}{$\begin{array}{l}\text { G3 - Desenvolvimento de perspectivas educacionais e } \\
\text { áreas de estudo na formação de professores indígenas }\end{array}$} & $05 \mathrm{~A}$ & \multirow{3}{*}{12} \\
\hline & $04 \mathrm{D}$ & \\
\hline & $03 \mathrm{~T}$ & \\
\hline \multirow{3}{*}{$\begin{array}{l}\text { G4 - Avaliação de Políticas Públicas e Documentos } \\
\text { Oficiais para a Formação de Professores Indígenas }\end{array}$} & $03 \mathrm{~A}$ & \multirow{3}{*}{08} \\
\hline & $02 \mathrm{D}$ & \\
\hline & $03 \mathrm{~T}$ & \\
\hline \multirow{3}{*}{$\begin{array}{l}\text { G5 - Formação de professor indígena para o Ensino de } \\
\text { CN }\end{array}$} & $01 \mathrm{~A}$ & \multirow{3}{*}{02} \\
\hline & $01 \mathrm{D}$ & \\
\hline & $00 \mathrm{~T}$ & \\
\hline \multicolumn{2}{|l|}{ Total } & 58 \\
\hline
\end{tabular}

Os trabalhos agrupados em G1 discutem acerca do ensino e da aprendizagem na formação de professores indígenas, mas, não abordam sobre o ensino de CN. Apresentam como foco de estudos: Temáticas na formação de professores indígenas (05, sendo 01 deles foca o corpo, 01 teatro, 01 território, 01 fotografia, 01 representação de gênero), Revitalização e papel da língua indígena na formação de professores indígenas (04), produção de materiais didáticos específicos (03), Educação Escolar Indígena (03), o papel da universidade na formação de professores indígenas 01, Processos Próprios de Aprendizagem (01), diálogo entre dois tipos de conhecimentos (02), o papel do livro 
didático no aprendizado (01), Identidade Docente (01) e modelos de avaliação da aprendizagem (01) (Quadro 3).

Quadro 03: Publicações que discutem o ensino e a aprendizagem na formação de professores indígenas organizados de acordo com o foco do estudo, encontradas dos sites da CAPES, BDTD e SCIELO, em 2017.

\begin{tabular}{|c|c|c|c|c|}
\hline \multicolumn{4}{|c|}{ G1- Ensino e aprendizagem na formação de professores indígenas } & Subtotais \\
\hline Temáticas na formação de professores indígenas & $02 \mathrm{~A}$ & $01 \mathrm{D}$ & $02 \mathrm{~T}$ & 05 \\
\hline $\begin{array}{l}\text { Revitalização e papel da língua indígena na formação de } \\
\text { professores indígenas }\end{array}$ & $01 \mathrm{~A}$ & $01 \mathrm{D}$ & $02 \mathrm{~T}$ & 04 \\
\hline $\begin{array}{l}\text { Educação Escolar Indígena (desafios, caminhos, novas } \\
\text { tendências) }\end{array}$ & $01 \mathrm{~A}$ & $00 \mathrm{D}$ & $02 \mathrm{~T}$ & 03 \\
\hline $\begin{array}{l}\text { Produção de materiais didáticos específicos nos cursos de } \\
\text { formação de professores }\end{array}$ & $01 \mathrm{~A}$ & $01 \mathrm{D}$ & $01 \mathrm{~T}$ & 03 \\
\hline Diálogo entre dois tipos de conhecimentos & $01 \mathrm{~A}$ & $00 \mathrm{D}$ & $01 \mathrm{~T}$ & 02 \\
\hline Identidade docente & $00 \mathrm{~A}$ & $00 \mathrm{D}$ & $01 \mathrm{~T}$ & 01 \\
\hline O papel do livro didático no aprendizado & $00 \mathrm{~A}$ & $01 \mathrm{D}$ & $00 \mathrm{~T}$ & 01 \\
\hline Processos próprios de aprendizagem & $01 \mathrm{~A}$ & $00 \mathrm{D}$ & $00 \mathrm{~T}$ & 01 \\
\hline Modelos de avaliação de aprendizagem & $00 \mathrm{~A}$ & $00 \mathrm{D}$ & $01 \mathrm{~T}$ & 01 \\
\hline $\begin{array}{l}\text { O papel da universidade na formação de professores } \\
\text { indígenas }\end{array}$ & $01 \mathrm{~A}$ & $00 \mathrm{D}$ & 00T & 01 \\
\hline Subtotais & $08 \mathrm{~A}$ & $04 \mathrm{D}$ & $10 T$ & 22 \\
\hline
\end{tabular}

A avaliação de Cursos de Formação de Professores Indígenas (G2) foi abordada com os seguintes focos de estudo (quadro 4): Abrangência de necessidades sócia cultural (01), Relação entre a formação de professores indígenas e sua prática pedagógica (01), Análise de uma experiência de formação de professores indígenas (12). Nenhum dos trabalhos abordam a área de CN.

Quadro 04: Trabalhos que discutem a avaliação de cursos de formação de professores indígenas, organizados de acordo com o foco de estudo, encontradas dos sites da CAPES, BDTD e SCIELO, em 2017.

\begin{tabular}{|c|c|c|c|c|}
\hline \multicolumn{4}{|l|}{ G2 - Avaliação de Cursos de Formação de Professores Indígenas } & Subtotais \\
\hline $\begin{array}{l}\text { Análise de uma experiência de formação de professores } \\
\text { indígenas }\end{array}$ & $03 \mathrm{~A}$ & $05 \mathrm{D}$ & $04 \mathrm{~T}$ & 12 \\
\hline Abrangência de necessidades sócio culturais & $00 \mathrm{~A}$ & $00 \mathrm{D}$ & $01 \mathrm{~T}$ & 01 \\
\hline $\begin{array}{l}\text { Relação entre a formação de professores indígenas e sua } \\
\text { prática pedagógica }\end{array}$ & $00 \mathrm{~A}$ & $01 \mathrm{D}$ & $00 \mathrm{~T}$ & 01 \\
\hline Subtotais & $03 \mathrm{~A}$ & $06 \mathrm{D}$ & $05 \mathrm{~T}$ & 14 \\
\hline
\end{tabular}

Os doze trabalhos agrupados no G3 (Desenvolvimento de perspectivas educacionais e áreas de estudo na formação de professores indígenas) discutem sobre o desenvolvimento de perspectivas educacionais e de áreas de estudo na formação de professores indígenas com os seguintes focos: 03 interculturalidade, 02 de linguística, 02 a formação do professor formador de professores indígenas, 01 na Educação Ambiental, 01 na Etnomatemática, 01 a formação continuada de professores, 01 a formação de professor de matemática e 01 a formação de professor de história (Quadro 05). Assim, os trabalhos agrupados em G3 não tratam sobre o ensino de $\mathrm{CN}$. 
Quadro 05: Publicações que discutem o desenvolvimento de perspectivas educacionais e áreas de estudo na formação de professores indígenas organizados de acordo com o foco do estudo, encontradas dos sites da CAPES, BDTD e SCIELO, em 2017.

\begin{tabular}{|c|c|c|c|c|}
\hline \multicolumn{4}{|c|}{$\begin{array}{l}\text { G3 - Desenvolvimento de perspectivas educacionais e áreas de estudo na } \\
\text { formação de professores indígenas }\end{array}$} & \multirow{2}{*}{$\begin{array}{c}\text { Subtotais } \\
03\end{array}$} \\
\hline Interculturalidade & $01 \mathrm{~A}$ & $02 \mathrm{D}$ & $00 \mathrm{~T}$ & \\
\hline Professor Formador de Professor Indígena & $00 \mathrm{~A}$ & $01 \mathrm{D}$ & $01 \mathrm{~T}$ & 02 \\
\hline Linguística & $02 \mathrm{~A}$ & $00 \mathrm{D}$ & $00 \mathrm{~T}$ & 02 \\
\hline Educação Ambiental & $01 \mathrm{~A}$ & $00 \mathrm{D}$ & $00 \mathrm{~T}$ & 01 \\
\hline Etnomatemática & $00 \mathrm{~A}$ & $01 \mathrm{D}$ & $00 \mathrm{~T}$ & 01 \\
\hline Formação Continuada & $00 \mathrm{~A}$ & $00 \mathrm{D}$ & $01 \mathrm{~T}$ & 01 \\
\hline Formação de professor de matemática & $00 \mathrm{~A}$ & $00 \mathrm{D}$ & $01 \mathrm{~T}$ & 01 \\
\hline Formação de professores de história & $01 \mathrm{~A}$ & $00 \mathrm{D}$ & $00 \mathrm{~T}$ & 01 \\
\hline Subtotais & $05 \mathrm{~A}$ & $04 \mathrm{D}$ & $03 \mathrm{~T}$ & 12 \\
\hline
\end{tabular}

Os trabalhos que compõem G4 também não abordam sobre o ensino de CN. Eles têm como foco de estudo: 06 as políticas públicas de Educação Escolar Indígena e formação de professores indígenas, 01 as políticas de formação em nível de magistério, 01 os percalços das políticas de formação de professores indígenas, 01 as políticas universitárias para a formação de professores indígenas, 01 os marcos curriculares dos "Referenciais para a formação de Professores Indígenas", 01 Situação atual da Formação de Professores Indígena (Quadro 06).

Quadro 06: Publicações que discutem a avaliação de políticas públicas e documentos oficiais para formação de professores indígenas organizados de acordo com o foco do estudo, encontradas dos sites da CAPES, BDTD e SCIELO, em 2017.

\begin{tabular}{|c|c|c|c|c|}
\hline \multicolumn{4}{|c|}{$\begin{array}{l}\text { G4 - Avaliação de políticas públicas e documentos oficiais para a Formação de } \\
\text { Professores Indígenas }\end{array}$} & \multirow{2}{*}{$\begin{array}{c}\text { Subtotais } \\
03\end{array}$} \\
\hline $\begin{array}{l}\text { Políticas públicas de Educação Escolar Indígena e } \\
\text { formação de professores indígenas }\end{array}$ & $01 \mathrm{~A}$ & $00 \mathrm{D}$ & $02 \mathrm{~T}$ & \\
\hline Políticas de formação em nível de magistério & $00 \mathrm{~A}$ & $01 \mathrm{D}$ & $00 \mathrm{~T}$ & 01 \\
\hline $\begin{array}{l}\text { Percalços das políticas de formação de professores } \\
\text { indígenas }\end{array}$ & $00 \mathrm{~A}$ & $00 \mathrm{D}$ & $01 \mathrm{~T}$ & 01 \\
\hline $\begin{array}{l}\text { Políticas universitárias para a formação de } \\
\text { professores indígenas }\end{array}$ & $01 \mathrm{~A}$ & $00 \mathrm{D}$ & $00 \mathrm{~T}$ & 01 \\
\hline $\begin{array}{l}\text { Marcos curriculares dos "Referenciais para a } \\
\text { formação de Professores Indígenas" }\end{array}$ & $00 \mathrm{~A}$ & $01 \mathrm{D}$ & $00 \mathrm{~T}$ & 01 \\
\hline Situação atual da Formação de Professores Indígena & $01 \mathrm{~A}$ & $00 \mathrm{D}$ & $00 \mathrm{~T}$ & 01 \\
\hline Subtotais & $03 \mathrm{~A}$ & $02 \mathrm{D}$ & $03 \mathrm{~T}$ & 08 \\
\hline
\end{tabular}

Como dito, 02 trabalhos, agrupados em G5 (01 artigo e 01 dissertação), discutem sobre a formação do professor indígena para o ensino de $\mathrm{CN}$ e foram desenvolvidos na Universidade Federal de Minas Gerais (UFMG). A dissertação, Tradição Maxakali e conhecimento científico: diferentes perspectivas para o conceito de transformação, tem como objetivo encontrar convergências e divergências entre a tradição Maxakali, grupo indígena de Minas Gerais e o pensamento científico. 
O artigo Entre o cristal e a chama: a natureza e o uso do conhecimento científico e dos saberes tradicionais numa disciplina do Curso de Formação Intercultural para Educadores Indígenas da Universidade Federal de Minas Gerais (FIEI/UFMG), apresenta como objetivo a discussão dos significados que envolvem a formação intercultural para educadores indígenas a partir das tensões decorrentes do encontro das diferentes culturas e da pluralidade de saberes em sala de aula.

Em síntese, 58 trabalhos, foram encontrados no levantamento acerca das publicações brasileiras sobre a formação de professores indígenas. Foram um total de 20 artigos, 21 teses e 17 dissertações. Se destacam nas produções científicas que abordam a formação de professores indígenas as instituições dos estados de SP, RS, AM e SC. Os trabalhos abordam na sua maioria sobre o ensino e aprendizagem na formação de professores indígenas, abordam ainda sobre o desenvolvimento de perspectivas educacionais e áreas de estudo na formação de professores indígenas e sobre a avaliação de políticas públicas, documentos oficiais e cursos de formação de professores indígenas.

Do total de publicações apenas 02 trabalhos apresentam relação direta com o tema da pesquisa, portanto, na formação de professores indígenas para o ensino de Ciências pouco se tem produzido. A seguir, apresentamos uma análise mais detalhada sobre o que anunciam essas publicações.

\section{Formação de professores indígenas nas publicações brasileiras: a área de ciências da natureza}

A dissertação de mestrado de Silveira (2010) intitulada: Tradição Maxakali e conhecimento científico: diferentes perspectivas para o conceito de transformação, foi desenvolvida na Universidade Federal de Minas Gerais, no âmbito do Programa de PósGraduação em Educação, Conhecimento e Inclusão Social. Apresenta uma discussão a respeito do ensino de ciências em escolas indígenas, a pesquisa procurou encontrar convergências e divergências entre a tradição Maxakali, grupo indígena de Minas Gerais e o pensamento científico no que se refere a cosmovisão desse povo e suas explicações para contextos cotidianos que envolvem o conceito científico denominado transformação química.

A pesquisa foi do tipo antropológica, teve abordagem qualitativa e desenvolveu-se em um dos módulos do curso de Formação Intercultural de Educadores Indígenas (FIEI), que ocorreu no primeiro semestre de 2009, no âmbito da disciplina -Temas Fundamentais em Química I- e a partir de visitas à aldeia. Teve como público alvo alunos da etnia Maxakali da comunidade de Aldeia Verde na área indígena Maxakali, que fica no município de Ladainha, em Minas Gerais. As fontes de dados foram as aulas do curso FIEl, além de observação e descrição de aspectos da cultura Maxakali através da convivência com a comunidade de Aldeia Verde.

As análises mostraram que há três tipos de explicações para as transformações químicas, dadas pelos Maxakali: a primeira, totalmente divergente com a ciência, considera a ação dos yãmĩy - espíritos - na ocorrência dos fenômenos. A segunda, mais convergente com o pensamento científico, está embasada em aspectos materiais e energéticos. 0 terceiro nos mostra situações em que os dois aspectos estão presentes. O aspecto objetivo, 
envolvendo apenas questões materiais e energéticas e o subjetivo, envolvendo a interação entre seres diferentes.

Silveira (2010) conclui, com base em Gasché (2004), que ensinar ciências nas escolas indígenas é um processo que exige um tratamento distinto daquele que utilizado nas salas de aula comuns. Possibilitar que os alunos indígenas transponham as fronteiras culturais que separam as duas formas de pensamento, tradicional e científico, requer do formador o reconhecimento de que são conhecimentos de origem diferente. Isso gera a necessidade de uma disposição em conhecer com certa profundidade o universo de seus alunos e língua falada por cada povo.

A autora explica ainda que a partir do estudo de determinadas atividades cotidianas indígenas, aluno e formador terão condições de estabelecer novas formas do exercício das faculdades psicológicas universais e de criar um entendimento mútuo ou intercompreensão intercultural. Entretanto, é importante conceber que mesmo quando estas atividades parecem adequadas à geração desse entendimento, é possível que existam outros fatores advindos do universo próprio indígena que irão interferir neste processo. Mesmo em casos em que conceitos científicos sejam reconhecidos pelos indígenas, é de se esperar que eles não os adotem como fim, já que nunca abandonarão suas explicações, constituintes de seu universo que é muito mais amplo que a ciência.

Valadares e Silveira (2016) no artigo: Entre o cristal e a chama: a natureza e o uso do conhecimento científico e dos saberes tradicionais numa disciplina do Curso de Formação Intercultural para Educadores Indígenas da Universidade Federal de Minas Gerais, discutem os significados que envolvem a formação intercultural para educadores indígenas a partir das tensões decorrentes do encontro das diferentes culturas e da pluralidade de saberes em sala de aula. As reflexões têm como panorama as questões em torno da função social da educação escolar indígena em suas articulações com as dinâmicas culturais em suas comunidades, considerando-se tanto a construção da educação básica nas aldeias quanto a formação docente na UFMG.

Foram analisadas algumas situações vivenciadas em sala de aula, em uma disciplina de formação de educadores indígenas, sob a metáfora do texto visto como chama ou cristal. $\mathrm{Na}$ aula, era desenvolvida a Disciplina Tópicos Especiais, com uma turma de estudantes indígenas da Faculdade de Educação (FAE) da UFMG. Especificamente, com a turma da habilitação Ciências da Vida e da Natureza (CVN) do Curso de Formação Intercultural para Educadores Indígenas, constituída por indígenas das etnias Xakriabá (MG) e Pataxó (BA e MG) (VALADARES; SILVEIRA, 2016).

As reflexões dos autores tiveram como pano de fundo as questões em torno da função social da educação escolar indígena em suas articulações com as dinâmicas culturais em suas comunidades. Essa discussão foi concomitante à tensão entre os conhecimentos acadêmicos e os conhecimentos tradicionais indígenas. Mostraram que, a cultura indígena está na escola quando o aluno indígena está presente, mas correndo o risco de passar despercebida, em função do predomínio de uma concepção cientificista do conhecimento (COHN, 2014). Valadares e Silveira (2016), constatam que a experiência permitiu uma melhor compreensão da realidade da educação escolar dos povos indígenas como contato cultural e não como folclore.

A pesquisas de Silveira (2010) e Valadares e Silveira (2016), trazem contribuições importantes acerca do ensino de Ciências na escola indígena e sobre o encontro de saberes tradicionais e científicos nas aulas de Ciências. Os trabalhos foram os únicos encontrados no 
mapeamento que tiveram proximidade com o tema, verificamos que ainda são tímidas as publicações nacionais acerca da formação de professores indígenas para o ensino de CN.

\section{Considerações finais}

O levantamento das publicações nacionais sobre a formação de professore indígenas em ensino de Ciências da Natureza é parte constituinte de um contexto maior, que se trata de uma pesquisa de mestrado sobre o tema, esse levantamento contribuiu para a compreensão dos trabalhos já realizados sobre a temática em estudo.

Nas buscas observamos que existe uma demanda grande de trabalhos relacionados à formação de professores indígenas, porém, quando filtramos a busca com os termos supracitados, sobre a formação de professores indígenas em ensino de Ciências da Natureza, verificamos uma diminuição considerável no quantitativo de trabalhos encontrados.

No total foram encontrados 58 trabalhos, 21 teses, 17 dissertações e 20 artigos. Esses trabalhos abordam temas variados, quando foram agrupados por tema abordado, verificamos que a maioria $(37,9 \%)$ tratam sobre ensino e aprendizagem na formação de professores indígenas, $24,1 \%$ sobre avaliação de cursos de formação de professores indígenas, 20,7\% abordam sobre o desenvolvimento de perspectivas educacionais e áreas de estudo na formação de professores indígenas, 13,9\% avaliam políticas públicas e documentos oficiais que tratam sobre o tema. Somente 2 trabalhos (1 artigo e 1 dissertação) que correspondem 3,4\% dos trabalhos encontrados, abordam acerca da formação de professores indígenas em Ciências da Natureza.

Como dito, a maior parte dos trabalhos foram publicados na região sudestes do Brasil, porém, ao fazer a leitura dos resumos, verificamos que muitos resultam de pesquisas realizadas na região norte por pesquisadores de outras regiões. Observamos ainda, a existência de pesquisadores da região norte, vinculados a instituições de outras regiões. Exemplo disso é a pesquisa mais ampla da qual esse estudo faz parte, que busca compreender a formação de professores indígenas em Ciências da Natureza na região Norte e está vinculada a Universidade Federal de Sergipe, pelo fato de no estado do Amapá, estado de origem da autora desse texto, não ter formação em cursos de pósgraduação na área de Ciências da Natureza.

Esse elevado número de estudos realizados por pesquisadores vinculados a instituições da região Sudeste e Sul, que têm a região Norte do Brasil como objeto de pesquisa, explicita que as pesquisas e a formação em Ciências da Natureza, ainda estão muito concentradas nas regiões sul e sudeste, com carência de programas de graduação e pósgraduação, nas outras regiões do país. Até mesmo nas da região Sudeste e Sul a produção relacionada à formação de professores indígenas em Ciências da Natureza é baixa, como dito, de todos os trabalhos encontrados, apenas dois tem relação direta com o tema.

Os dois trabalhos encontrados no mapeamento, que abordam acerca da formação de professores indígenas em Ciências da Natureza, foram desenvolvidos na Faculdade de Educação da UFMG no Curso de Formação de Educadores Indígenas, em turmas da habilitação em Ciências da Vida e da Natureza. Apesar da semelhança nos nomes, foram produzidos por autores diferentes, o primeiro em 2010 e o segundo em 2016. Ambos discutem sobre tensões e conflitos decorrentes do encontro entre saberes indígenas e os saberes provenientes da Ciência Moderna, assim como da diversidade cultural presente na 
formação de professores indígenas. São questões importantes para o desenvolvimento do ensino de Ciências da Natureza e para a efetivação da política nacional de Educação Escolar Indígena.

Compreendemos que a formação de professores indígenas, em Ciências da Natureza, exerce papel chave, para que a área de Ensino de Ciências possa contribuir com os projetos de autossustentação e autodeterminação dos povos indígenas, essa é uma demanda do movimento nacional de Educação Escolar Indígena. Esse pensamento do movimento indígena recebe acolhida nos Referenciais para Formação de Professores Indígenas (BRASIL, 2002) e nas Diretrizes Curriculares Nacionais para Formação de Professores Indígenas em cursos de Educação Superior e de Ensino Médio (BRASIL, 2015).

Representantes do movimento da Educação Escolar Indígena, que são colaboradores dessa pesquisa, falam da necessidade do ensino de CN na escola indígena, ser pautado nas aspirações de cada povo e comunidade. Para isso, a formação de professores indígenas deve ser específica e diferenciada, abrangendo questões que visem além da capacitação técnica, a formação política do futuro professor, com a participação de sábios e lideranças indígenas, e pautada no princípio da interculturalidade.

Verificamos que essa formação, em Ciências da Natureza, tem sido feita em todo país, pelos cursos de Licenciaturas interculturais - como são denominados os cursos de licenciatura para a formação de professores indígenas - que são cursos de formação geral, que ofertam entre outras, a habilitação em Ciências da Natureza. Como dito, os únicos dois trabalhos encontrados no levantamento, foram resultados de atividades realizadas no âmbito de um curso de licenciatura Intercultural da UFMG.

O campo de formação de professores indígenas é novo no Brasil e, mais especificamente, as investigações sobre a formação de professores indígenas para o ensino de $C N$, esse mapeamento das publicações nacionais, favorece a compreensão do que anunciam os trabalhos já realizados sobre a temática em estudo e dá indícios de como vem acontecendo esse processo formativo.

\section{Referências}

BRASIL. Constituição da República Federativa do Brasil. Brasília: 1988.

BRASIL. Ministério da Educação. Referencial Curricular Nacional Para as Escolas Indígenas. Brasília: MEC/SEF, 2002b.

BRASIL. Ministério da Educação. Referenciais para a formação de professores Indígenas. Brasília: MEC, 2002

BRASIL. Ministério da Educação. Resolução nº 1, de 7 de janeiro de 2015. Diretrizes Curriculares Nacionais para a Formação de Professores Indígenas em cursos de Educação Superior e de Ensino Médio e dá outras providências. Disponível em: http://portal.mec.gov.br/index.php?option=com_docman\&view=download\&alias=16870 res-cne-cp-001-07012015\&ltemid=30192. Acesso em: 01 jun 2018.. 
CAVALCANTE, Lucíola Inês Pessoa. Formação de Professores na perspectiva do movimento dos professores indígenas da Amazônia. In: Revista Brasileira de Educação, N. 22, p. 14-24. 2003. Disponível em: <www.scielo.br/pdf/rbedu/n22/n22a03>. Acesso em: 19 maio 2017.

FERNANDES, Dulcilene Rodrigues. Formação de professores indígenas: um rito de passagem? Cuiabá: EDUFMT, 2009.

GRUPIONI, Luís Donisete Benzi. Experiência e desafios na formação de professores indígenas no Brasil. In: Em Aberto. Brasília: INEP, v. 20, n. 76, p. 13-18. 2003. Disponível em: <aberto.inep.gov.br/index.php/emaberto/issue/download/238/78. Acesso em: 19 maio 2017.

LOPES, Edinéia Tavares. Ensino-Aprendizagem de Química na Educação Escolar Indígena: O Uso do Livro Didático de Química em um contexto Bakairi. Química Nova na Escola, São Paulo, vol. 37, n 4, p. 249-256, nov. 2015. Disponível em: <http://qnesc.sbq.org.br/online/qnesc37_4/03-EA-12-14.pdf>.

LOPES, Edinéia Tavares; BRITO, Edson Machado de Brito; ROSA, Silvana da Costa Santa; JESUS, Yasmin Lima de Jesus. Justiça social: utopias e realidades na elaboração da e na Educação Escolar Indígena. In: OLIVEIRA, Ilzver de Matos; SOUZA, Otavio Augusto Reis de; ANDRADE, Fábio Santos de Andrade (Orgs.). Movimentos Sociais, Justiça e sóciobiodiversidade: Pesquisas Contemporâneas. Curitiba: Editora CRV, 2017.

SILVEIRA, Katia Pedroso. Tradição Maxakali e o conhecimento científico: diferentes perspectivas para o conceito de transformação. Dissertação (Mestrado). UFMG: Belo Horizonte, 2010. Disponível em:

<http://www.bibliotecadigital.ufmg.br/dspace/handle/1843/BUDB-8C7MSH>. Acesso em: 15 maio 2017.

VALADARES, Juarez Melgaço; SILVEIRA, Célio da. Entre o cristal e a chama: a natureza e o uso do conhecimento científico e dos saberes tradicionais numa disciplina do Curso de Formação Intercultural para Educadores Indígenas da Universidade Federal de Minas Gerais (FIEI/UFMG). Ciências e Educação, Bauru, v. 22, n. 2, p. 541-553. 2016. Disponível em: <http://dx.doi.org/10.1590/1516-731320160020016>. Acesso em: 04 jul. 2017. 